\title{
Análisis cuarititativo de cuarzo-alfa en mezclas de cemento portland anhidro y calcita sintética por difracción de rayos $X$ con equipo Philips $\mathrm{PW}-1700$
}

J. L. SAGRERA-MORENO, Dr. en Ciencias Quimicas y Ledo. en Ciencias de la Información
(IETCC/CSIC)

\section{$R E S U M E N$}

En el presente articulo se explican los pasos necesarios para realizar un análisis cuantitativo de cuarzo-alfa aplicando la técnica de difracción de rayos $X$ en mezclas artificiales de cemento portland anhidro y calcita sintética, cuyos contenidos son conocidos.

\section{$S U M M A R Y$}

In this article there are explained the necessary' steps to make up a quantitative analysis of alpha-quartz applying the technique of rays $X$ diffraction, in artificial mixtures of anhydrous portland cement and synthetic calcite, which contents are known.

\section{PREPARACION DE LAS MUESTRAS}

Se han preparado tres muestras, denominadas M2, M2MAS1 y M2MAS2 cuya composición es la siguiente:

$$
\begin{aligned}
& \text { M2 }=5 \mathrm{~g} \text { cemento }+2,5 \mathrm{~g} \text { calcita }+2,5 \mathrm{~g} \text { alfa- } \mathrm{SiO}_{2} \\
& \text { M2MAS1 }=5 \mathrm{~g} \text { cemento }+2,5 \mathrm{~g} \text { calcita }+3,5 \mathrm{~g} \text { alfa- } \mathrm{SiO}_{2} \\
& \text { M2MAS2 }=5 \mathrm{~g} \text { cemento }+2,5 \mathrm{~g} \text { calcita }+4,5 \mathrm{~g} \text { alfa- } \mathrm{SiO}_{2}
\end{aligned}
$$

por tanto las concentraciones de alfa- $\mathrm{SiO}_{2}$ en cada muestra son las siguientes:

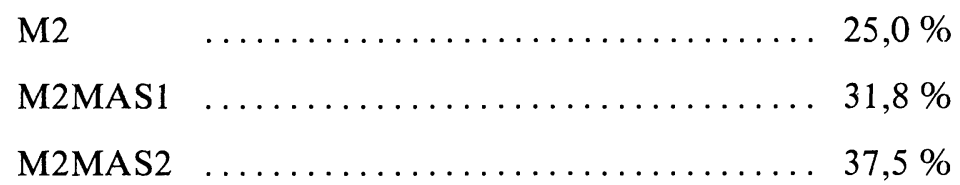

En los compuestos de cada muestra, tanto el cemento como la calcita, fueron desecados a $105^{\circ} \mathrm{C}$ durante 24 horas habiéndose previamente porfirizado la calcita en mortero de ágata.

Los tres compuestos de cada muestra fueron homogeneizados mediante un molino vibratorio marca SPECAMILL tipo 6.000 de la firma SPECAC (Londres) durante 15 minutos, utilizando un recipiente de CELOTEX cilindrico, de $47 \mathrm{~mm}$ de altura y $13 \mathrm{~mm}$ de diámetro interior, cuyas bases fueron cerradas mediante sendos tapones de goma virgen, impidiendo la salida y consiguiente pérdida de muestra.

Las primeras pruebas se hicieron utilizando un recipiente de vidrio que presentó grietas a causa 
de la vibración. También se hicieron pruebas utilizando tubo de plástico fino, obteniéndose igual resultado.

Para la homogeneización de las muestras se introdujeron, en el recipiente de CELOTEX, tres bolas de acero de $8 \mathrm{~mm}$ de diámetro. La determinación de la amplitud de la vibración se realizó experimentalmente, fijándose cuando se consigue que sea máxima.

Las muestras, una vez preparadas, se prensaron en una máquina HERZOG (modelo HDIFP 1982) utilizando los portamuestras que tiene el equipo de difracción de rayos $\mathrm{X}$ y siguiendo la normativa especificada en (1). El efecto de prensado de la muestra en polvo evita su posible destrucción cuando, al ser analizada en el equipo, se utiliza el sistema giratorio de muestras, factor éste que proporciona una integración de la superficie barrida por el haz de rayos $\mathrm{X}$ y, por tanto, una mejor representación de la muestra analizada.

\section{COMPOSICION DEL EQUIPO UTILIZADO}

Se ha empleado un equipo automático de difracción de rayos $\mathrm{X}$, marca Philips, compuesto de las siguientes unidades:

- Generador de alta tensión de $4 \mathrm{~kW}, \mathrm{PW}-1730$.

- Rendija automática de divergencia, PW-1386/50.

- Monocromador de grafito, PW-1743/00.

- Portamuestras automático para 42 muestras, PW-1780.

- Unidad de refrigeración automática, DM-6000.

- Software analítico Philips APD-1700 (V.S.2.0).

- Miniordenador DIGITAL, modelo PDP 11/24 de 256 Kbytes.

- Teleimpresora DIGITAL, modelo LA-120.

- Terminal gráfico Hewlett-Packard, modelo 2648-A.

- Impresora gráfica Hewlett-Packard, modelo 2631-G.

- Prensa semiautomática Herzog, modelo HDIFP.

- Portamuestras modificados por el IETcc (diámetro $=20$ milimetros).

\section{CONDICIONES DE TRABAJO}

Las condiciones instrumentales utilizadas son las siguientes:

- Generador trabajando a $40 \mathrm{kV}$ y $50 \mathrm{~mA}$.

- Tubo con ánodo de cobre.

- Avance del paso de goniómetro $0,020^{\circ}$.

- Tiempo de recuento en cada paso 0,80 segundos.

- Rango del registro desde $2 \theta=15^{\circ}$ a $2 \theta=25^{\circ}$.

- Monocromador de grafito (elimina lineas K-beta).

(1) SAGRERA-MORENO, J. L. (1985): Preparación de muestras para análisis por difracción de rayos X con el equipo Philips PW-1700. Materiales de Construcción. Vol. 34, n. 195, julio/agosto/septiembre 1984. 
- Rendija automática de divergencia.

- Condiciones de la discriminación por altura de impulsos, niveles máximo $=70 \mathrm{~V}$, míni$\mathrm{mo}=35 \mathrm{~V}$.

- Velocidad del registrador $=20$.

- Número de cuentas máximo dentro de escala de papel $2 \mathrm{E} 3=2.000$ cuentas.

- Constante de tiempo automática (S).

\section{ELECCION DE LA REFLEXION DEL CUARZO-ALFA}

Realizado un registro en las proximidades del pico, con valor $2 \theta=20,9^{\circ}$, se comprobó que los compuestos cristalinos de la muestra no presentaban ninguna reflexión en dicho lugar; por tanto, se tomó esta reflexión para el cálculo de la concentración de cuarzo-alfa en las muestras estudiadas (figura 1).

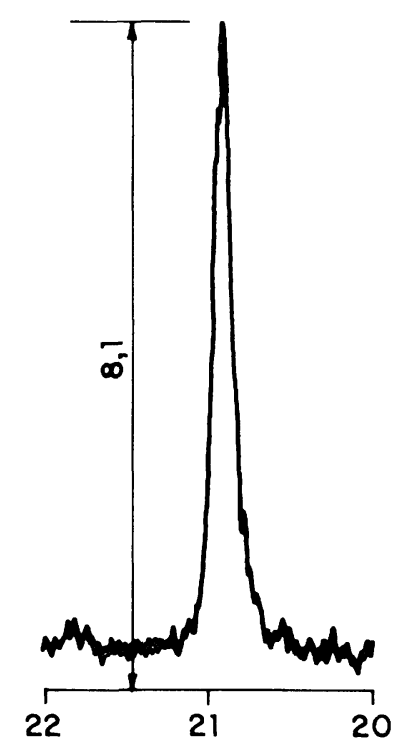

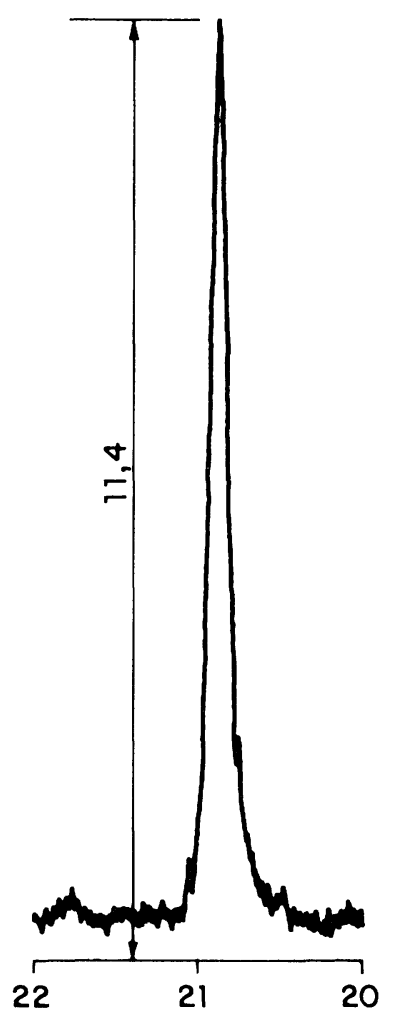

Fig. 1.

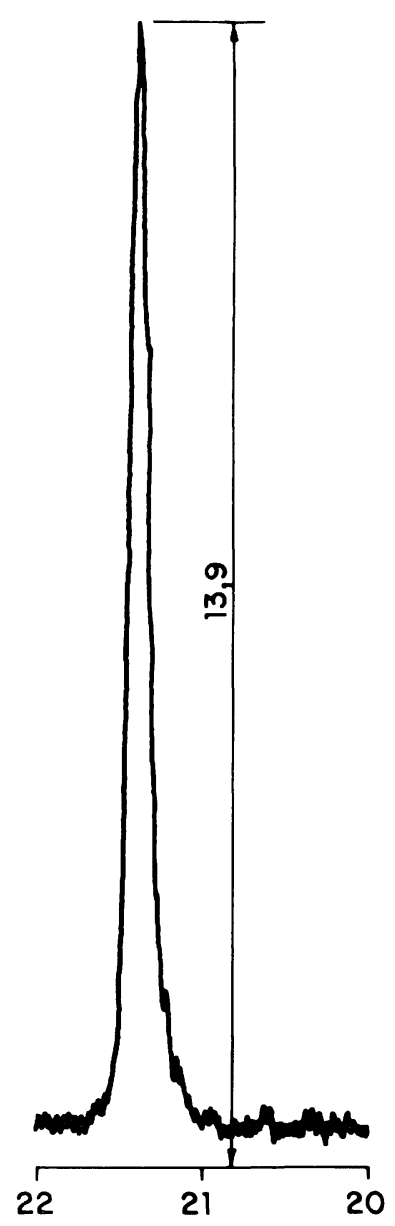




\begin{tabular}{|c|c|}
\hline Muestras & Angulos de reflexión en $2 \theta$ \\
\hline M2 & $15,6100-20,9175-21,800-23,0625-24,4175$ \\
\hline M2MAS1 & $20,8775-23,0725-24,3500$ \\
\hline M2MAS2 & $20,9175-23,0775-24,4250$ \\
\hline
\end{tabular}

Los valores correspondientes al cuarzo-alfa son para cada muestra:

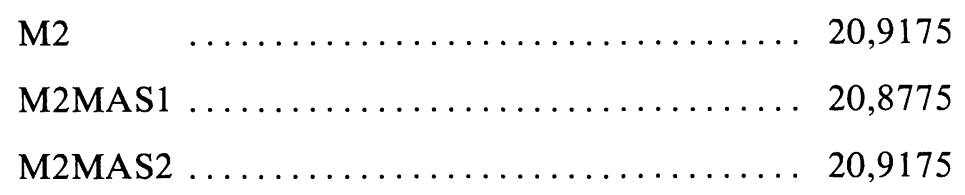

Las pequeñas diferencias en los valores de $2 \theta$ para las reflexiones del cuarzo-alfa, en cada muestra, son debidas a la suma de error en preparación de muestra y error mecánico del goniómetro del equipo.

Tomando en cada uno de los listados de picos, correspondientes a las muestras registradas, los valores de cuentas en el pico y en el fondo (Peack y Backg), y efectuando la resta entre uno y otro, obtenemos el valor de cuentas netas obtenidas en los valores correspondientes al pico del cuarzo-alfa en cada muestra, es decir:

\begin{tabular}{|c|c|c|c|c|}
\hline Muestra & Angulo & Pico & Fondo & Neto \\
\hline M2 & 20,9175 & 480 & 37 & 443 \\
\hline M2MAS1 & 20,8775 & 666 & 38 & 628 \\
\hline M2MAS2 & 20,9175 & 841 & 36 & 805 \\
\hline
\end{tabular}

Por tanto, estos valores obtenidos directamente del listado de picos de cada muestra, nos proporciona un número proporcional al contenido de cuarzo-alfa en cada muestra.

\section{Cálculos}

Llamando $\mathrm{X}$ al contenido de cuarzo-alfa de la muestra M2 podemos decir:

$$
\begin{aligned}
& X+2 \text { g cuarzo-alfa } \ldots \ldots \ldots \ldots \ldots \ldots \ldots .605 \text { cuentas } \\
& X+1 \text { g cuarzo-alfa } \ldots \ldots \ldots \ldots \ldots \ldots \ldots .628 \text { cuentas }
\end{aligned}
$$

Resta

$1 \mathrm{~g}$ cuarzo-alfa

177 cuentas

Luego

$$
\begin{aligned}
& 177 \text { cuentas } \ldots \ldots \ldots \ldots \ldots \ldots \ldots \ldots \ldots \ldots \ldots \ldots \ldots \ldots \ldots \ldots \ldots \ldots \ldots \\
& 443 \text { cuentas } \ldots \ldots \ldots \ldots \ldots \ldots \\
& X=\frac{443 \times 1}{177}=2,5 \text { gramos cuarzo-alfa }
\end{aligned}
$$

que coincide con la cantidad de cuarzo-alfa existente en la muestra M2. 
Haciendo los cálculos en tanto por ciento obtenemos:

805 cuentas $\ldots \ldots \ldots \ldots .(x+20) \%$ de cuarzo-alfa

628 cuentas $\ldots \ldots \ldots \ldots .(x+10) \%$ de cuarzo alfa

Resta

177 cuentas .....10 \% de cuarzo-alfa

443 cuentas ...... y \% de cuarzo-alfa en muestra inicial

$$
\mathrm{y}=\frac{443 \times 10}{177}=\frac{4.430}{177}=25,03 \%
$$

resultado que coincide con el contenido de cuarzo-alfa en la muestra sintética M2 (5 g cemento $+2,5$ g calcita $+2,5$ g cuarzo-alfa).

Tomando en los difractogramas las alturas de cada uno de los picos representativos del cuarzoalfa, puede comprobarse que el resultado no es tan cierto como utilizando el número de cuentas netas.

Las proporciones que pueden establecerse entre cuentas netas y alturas de los picos correspondientes es la siguiente:

$$
\frac{805 \text { cuentas }}{13,9 \mathrm{~cm}}=\frac{628 \text { cuentas }}{11,4 \mathrm{~cm}}=\frac{443 \text { cuentas }}{8,1 \mathrm{~cm}}
$$

Los cocientes respectivos son:

$$
57,91 \neq 55,09 \neq 54,69
$$

resultados desiguales entre sí, ya que la representación gráfica no es tan válida como el recuento de impulsos en la reflexión correspondiente al compuesto cristalino analizado.

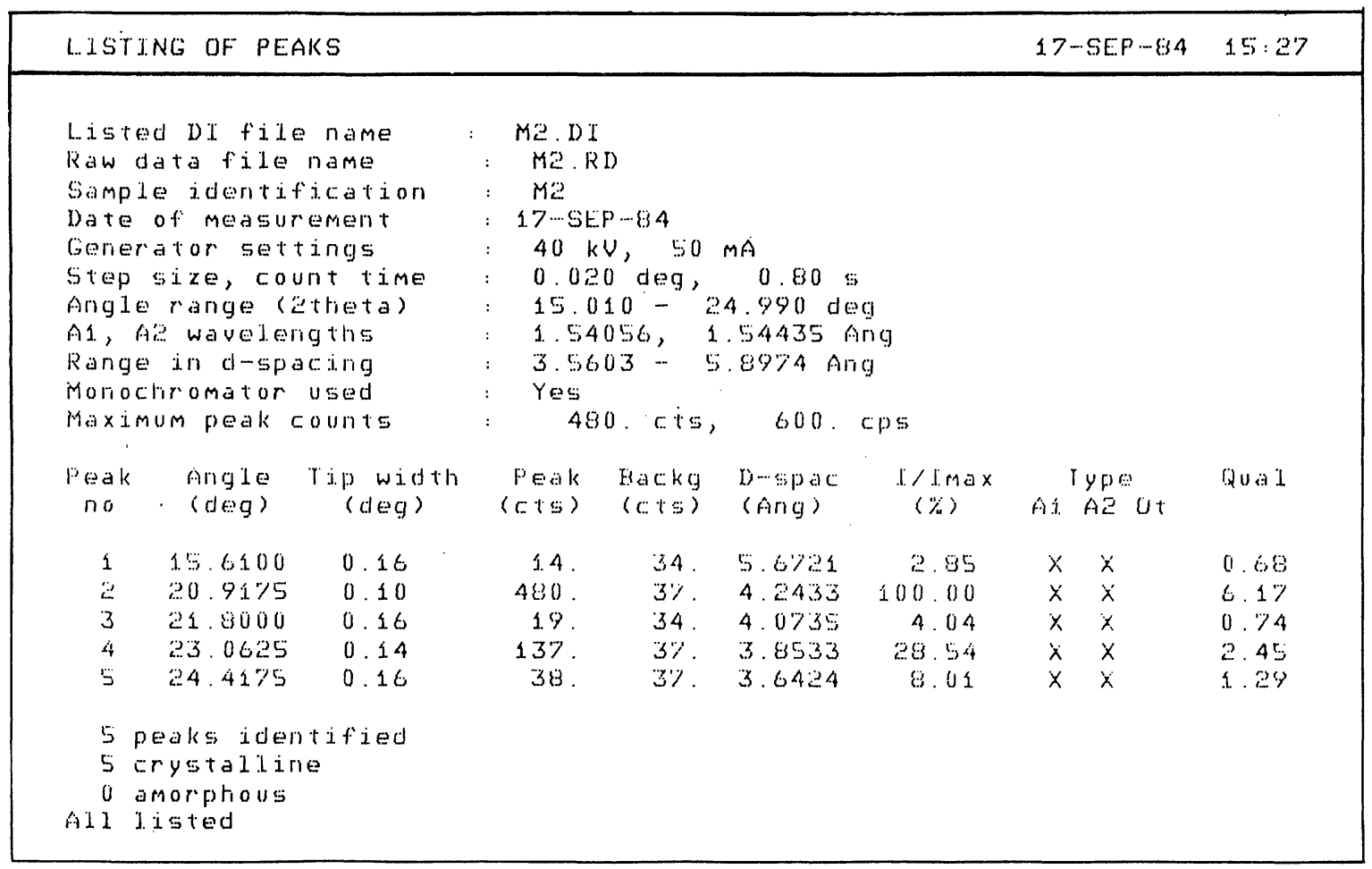




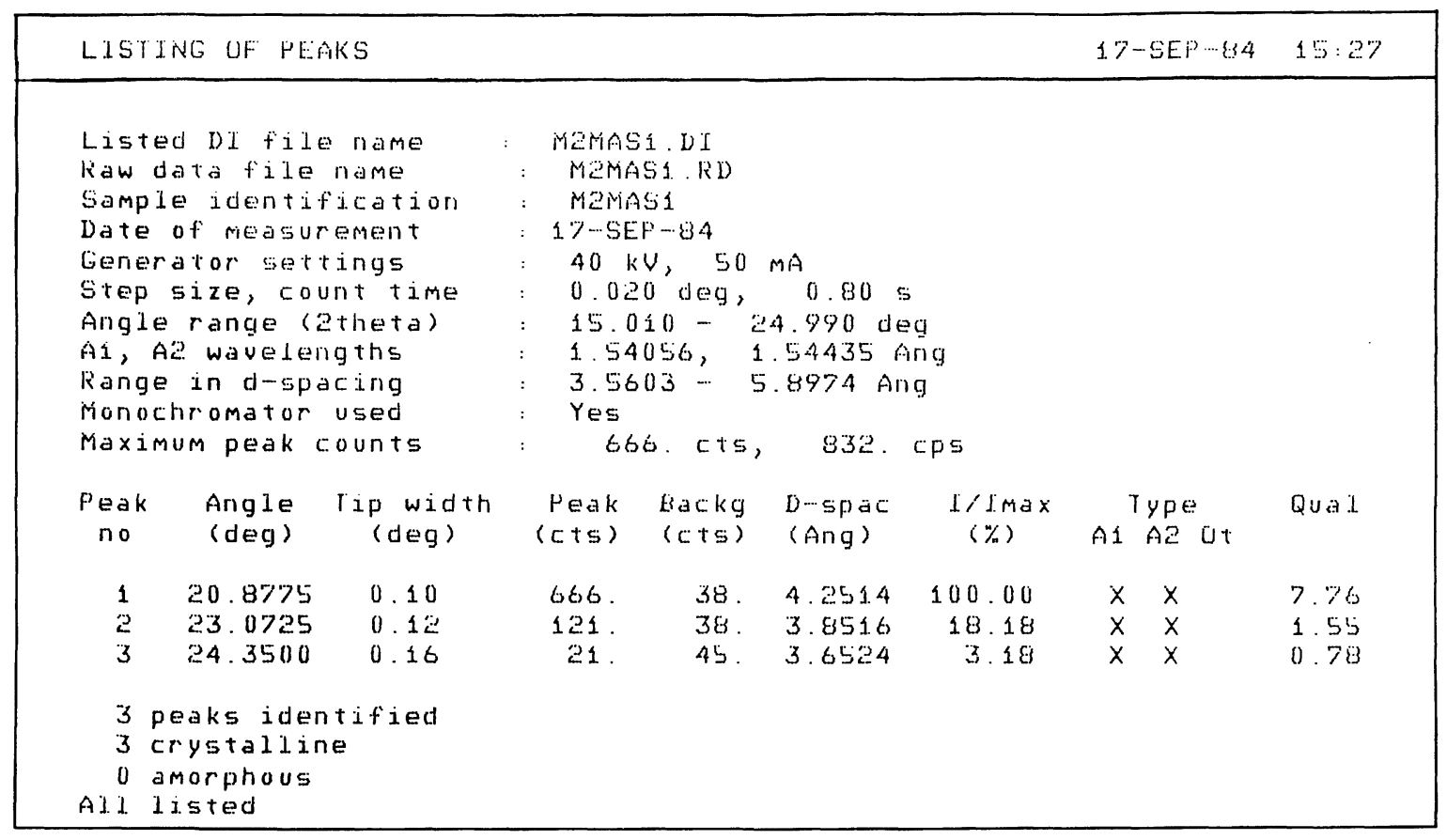

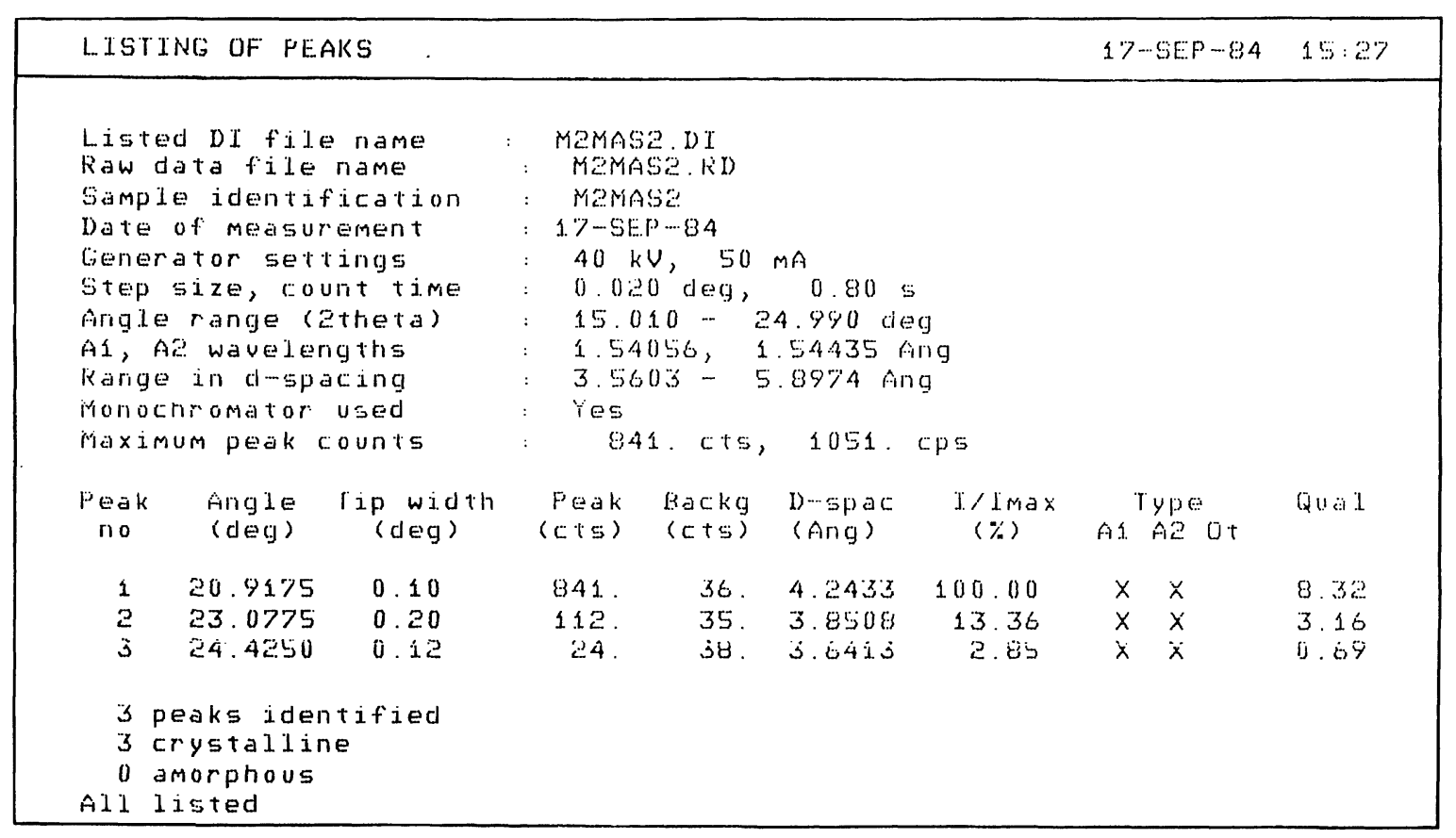

En sucesivos artículos se irán explicando paso a paso las técnicas para, aplicando diferentes métodos, según el caso, realizar análisis cuantitativos por difracción de rayos $\mathrm{X}$. 